\title{
Improved Polynomial Fuzzy Modeling and Controller with Stability Analysis for Nonlinear Dynamical Systems
}

\author{
Hamed Kharrati, 1, 2 Sohrab Khanmohammadi, 1 \\ Witold Pedrycz, ${ }^{2}$ and Ghasem Alizadeh ${ }^{1}$ \\ ${ }^{1}$ Faculty of Electrical and Computer Engineering, University of Tabriz, \\ P.O. Box 5166616471, Tabriz, Iran \\ ${ }^{2}$ Department of Electrical and Computer Engineering, University of Alberta, Edmonton, \\ $A B$, Canada T6G 2 V4
}

Correspondence should be addressed to Hamed Kharrati, kharrati@ualberta.ca

Received 25 May 2012; Revised 30 September 2012; Accepted 4 October 2012

Academic Editor: Mohammed Chadli

Copyright (C) 2012 Hamed Kharrati et al. This is an open access article distributed under the Creative Commons Attribution License, which permits unrestricted use, distribution, and reproduction in any medium, provided the original work is properly cited.

\begin{abstract}
This study presents an improved model and controller for nonlinear plants using polynomial fuzzy model-based (FMB) systems. To minimize mismatch between the polynomial fuzzy model and nonlinear plant, the suitable parameters of membership functions are determined in a systematic way. Defining an appropriate fitness function and utilizing Taylor series expansion, a genetic algorithm (GA) is used to form the shape of membership functions in polynomial forms, which are afterwards used in fuzzy modeling. To validate the model, a controller based on proposed polynomial fuzzy systems is designed and then applied to both original nonlinear plant and fuzzy model for comparison. Additionally, stability analysis for the proposed polynomial FMB control system is investigated employing Lyapunov theory and a sum of squares (SOS) approach. Moreover, the form of the membership functions is considered in stability analysis. The SOS-based stability conditions are attained using SOSTOOLS. Simulation results are also given to demonstrate the effectiveness of the proposed method.
\end{abstract}

\section{Introduction}

Due to general and systematic platforms to deal with nonlinear control problems and various applications, research on Fuzzy Model-Based (FMB) control methodology has received significant attention in the last decade [1-3]. The fuzzy logic strategy is one of the most popular tools for modeling and control of ill-defined and complex nonlinear plants. This line of research is motivated by the attractive properties of fuzzy logic control including robustness to disturbances, model parameter uncertainties, and sensor noise. Preliminary 
works were done based on expert knowledge and data-driven rule/knowledge extraction approaches, where the system dynamics is identified using data [4-11]. Numerous FMB techniques have been applied to modeling, control, and stability analysis of nonlinear dynamical plants. The Lyapunov stability theory is the most common approach which is used to derive stability conditions for nonlinear plants under the FMB control systems [12-17]. However, involving fuzzy models instead of original plant makes some mismatch concerns, which should be considered in fuzzy control. Hence, achieving sound results acquired in the FMB control framework is the fundamental objective of this study.

Nowadays, increasing trend in theoretical developments of the control system stability has encouraged the improvement of FMB systems [18-20]. The Takagi-Sugeno (T-S) fuzzy model is the most commonly considered architecture used for nonlinear control systems modeling and their stability analysis via linear matrix inequalities (LMIs) [21]. A plethora of researches exist in literatures investigating on various strategies to develop and facilitate the performance design and stability analysis of the generic T-S approach. The initial attempts with this regard were proposed in [22, 23]. Then, the well-known parallel distributed compensation (PDC) procedure was presented in [24]. In this method, the fuzzy controller shares the same membership functions as those of the T-S fuzzy model. Based on the PDC technique, various relaxed LMI-based stability conditions were derived in [25-28]. The advantage of these proposed researches is that the obtained stability conditions are independent of membership functions parameters and consequently stability analysis is facilitated. Although sharing the same premise variables in both fuzzy plant model and controller make stability analysis easier, and the robustness property of FMB controller is scarified. This arises from the fact that the parameters of membership functions must be known entails and known fuzzy plant model. On the other hand, fuzzy models are generally employed in the case, where the model structure and parameters are unknown [29-31]. It was revealed in [32-35] that the role of membership functions in FMB control system is obviously effective, and obtained stability conditions without their consideration are very conservative. Thus, the FMB controller under such design constraint is not applicable for dealing with nonlinear systems under parameter uncertainties. A method of online modeling and control via an adaptive T-S fuzzy-neural model for a class of uncertain nonlinear systems was developed in [36]. In [37], a robust adaptive fuzzy modeling and control approach were proposed for a class of multi-input-multioutput (MIMO) nonlinear systems using the approximation property of the fuzzy logic systems and the back-stepping technique. A robust static output controller for discrete time T-S fuzzy models with uncertainties was also proposed in [38]. In this work, descriptor procedure employed to solve stabilization problem based on strict LMIs structure. The works in [39-42] involved different fuzzy model reference methods for modeling and control problem of nonlinear systems based on T-S fuzzy models. Indirect/direct model reference fuzzy controllers were introduced in these studies. Most of the aforementioned efforts on adaptive T-S fuzzy models were considered for only a certain class of nonlinear plants. For instance, the proposed procedures are for a first-order nonlinear dynamical plant. Furthermore, it is not possible to consider every desirable reference model. Due to linear subsystems in T-S fuzzy model and the limited number of fuzzy rules, there is an unavoidable mismatch between the original nonlinear plant and the fuzzy model. Besides, because of nonsuitable platform, these works lacked comprehensive stability analysis on the resultant closed-loop system. In fact, the full information of membership functions are not taken into stability conditions due to limitation 
of existing LMI toolbox which can only find solution for a finite setup of LMIs. Therefore, formulation of continuous membership functions in LMI terms is not practically possible. To overcome the aforementioned drawbacks of LMI-based techniques, a new framework has been recently introduced based on a sum of squares (SOS) decomposition for modeling and control of nonlinear systems [43]. As the polynomial subsystems are allowed in the consequent part of fuzzy rules, a wide range of nonlinear plants could be represented more precisely than traditional T-S technique, and the number of fuzzy rules can be reduced, as well. In this paper, a proposal is made to achieve an improved polynomial fuzzy modeling and design a polynomial fuzzy controller using PDC strategy for nonlinear systems. Also, the proposed modeling provides a suitable platform for a comprehensive stability analysis of obtained closed-loop FMB control system.

More specifically, the main contribution of this paper is thorough investigations of accurate fuzzy model for nonlinear dynamical plants based on PDC technique and polynomial fuzzy systems via tuning the membership functions. Employing Taylor series expansion, the grades of membership functions are extracted in the form of polynomials in corresponding sub-regions which establishe a platform for a comprehensive stability analysis. As the SOS technique will be subsequently employed for stability analysis, it is worthwhile to represent the membership functions in the form of polynomials. Defining an appropriate fitness function, genetic algorithm (GA) is used to determine the coefficients of polynomial membership grades to minimize the mismatch error between the original nonlinear plant and the polynomial fuzzy model. In purpose of illustration, the nonlinear plant dynamics are assumed to be known in this paper. To form the FMB control system, a controller is designed based on the fuzzy PDC concept to close the feedback loop. The proposed fuzzy modeling and controller provide a structure to utilize information about membership functions into the stability analysis and derive the SOS-based stability conditions for the polynomial FMB control system. The SOS-based stability conditions can be numerically attained by SOSTOOLS (a third-party Matlab toolbox).

The remainder of the paper is organized as follows. Section 2 details the improved FMB control system divided to four subsections. Section 2.1 reviews the polynomial fuzzy modeling for nonlinear systems. Model improvement based on Taylor series expansion is presented in Section 2.2 using GA. Based on the PDC technique, a polynomial fuzzy controller is constructed for the improved polynomial fuzzy model in Section 2.3. Finally, Section 2.4 proposes the SOS-based stability conditions of designed FMB control system considering the Lyapunov stability theory. In Section 3, two simulation examples are given to demonstrate the feasibility of the developed technique. A comparing example at the end of this section is presented in order to show the improvements of the proposed methodology in this paper in comparison with previous fuzzy PDC controllers. We make a discussion in Section 4 about the priority of our proposed approach compared with the basic polynomial fuzzy method. Conclusions are summarized in Section 5.

\section{Improved FMB Control System}

In what follows, we present a methodology, which leads to the improved FMB control system including stability analysis. The computational approach used in this paper relies on the Taylor series expansion, GA, and SOS decomposition of multivariable polynomials. 


\subsection{Polynomial Fuzzy Modeling}

In order to introduce polynomial fuzzy modeling, consider the following nonlinear model expressed in state space:

$$
\dot{\mathbf{x}}(t)=\mathbf{f}(x(t), \mathbf{u})
$$

where $\mathbf{f}$ is a known nonlinear function, $\mathbf{x}(t)=\left[x_{1}(t), \ldots, x_{n}(t)\right]^{T}$ and $\mathbf{u}(t)=\left[u_{1}(t), \ldots\right.$, $\left.u_{m}(t)\right]^{T}$ are the state and input vectors, respectively, and $t$ denotes time. Based on the sector nonlinearity concept, the following polynomial fuzzy model describes the dynamics of nonlinear model in (2.1) [43,44].

Model Rule $i$ :

$$
\begin{aligned}
& \text { if } z_{1}(\mathbf{x}(t)) \text { is } M_{1}^{i} \text { and } \ldots \text { and } z_{\tau}(\mathbf{x}(t)) \text { is } M_{l^{\prime}}^{i} \\
& \text { then } \dot{\mathbf{x}}(t)=\mathbf{A}_{i}(\mathbf{x}(t)) \boldsymbol{X}(\mathbf{x}(t))+\mathbf{B}_{i}(\mathbf{x}(t)) \mathbf{u}(t), \quad i=1,2, \ldots, r,
\end{aligned}
$$

where $i=1, \ldots, r$. Here, $z_{\gamma}(\mathbf{x}(t))(h=1, \ldots, l)$ represents the premise variables. $M_{h}^{i}$ denotes the membership function associated with the $i$ th rule of the model and $h$ th premise variable. The number of fuzzy rules is denoted by $r$. Each $z_{h}(\mathbf{x}(t))$ is a measurable time-varying quantity that may be states, measurable external variables, and/or time. $\mathbf{A}_{i}$ and $\mathbf{B}_{i}$ are the polynomial matrices in $\mathbf{x}(t) \cdot \mathcal{X}(\mathbf{x}(t)) \in R^{N}$ is a $N \times 1$ column vector whose elements are all monomials in $\mathbf{x}(t)$.

Definition 2.1 (see [44]). A monomial in $\mathbf{x}(t)$ is a function of the form $x_{1}^{\alpha_{1}} x_{2}^{\alpha_{2}} \cdots x_{n}^{\alpha_{n}}$, where $\alpha_{1}, \alpha_{2}, \ldots, \alpha_{n}$ are nonnegative integers. The degree of monomial is given by $\alpha_{1}+\alpha_{2}+\cdots+\alpha_{n}$.

Hence, $\mathbf{A}_{i}\left(\mathbf{x}(t) \mathcal{X}(\mathbf{x}(t))+\mathbf{B}_{i}(\mathbf{x}(t)) \mathbf{u}(t)\right.$ is a polynomial vector. Therefore, each consequent part of the polynomial fuzzy model (2.2) is a polynomial.

The overall system dynamics is computed by combining the fuzzy rules in (2.2) (subsystems) as follows:

$$
\dot{\widehat{\mathbf{x}}}(t)=\sum_{i=1}^{r} p_{i}(\mathbf{x}(t))\left\{\mathbf{A}_{i}(\mathbf{x}(t)) \boldsymbol{X}(\mathbf{x}(t))+\mathbf{B}_{i}(\mathbf{x}(t)) \mathbf{u}(t)\right\}
$$

where $\dot{\hat{\mathbf{x}}}(t)$ is the estimated dynamics of the original nonlinear plant in (2.1) represented by the polynomial fuzzy model. The expressions

$$
p_{i}(\mathbf{x}(t))=\frac{\prod_{h=1}^{l} M_{h}^{i}\left(z_{h}(\mathbf{x}(t))\right)}{\sum_{k=1}^{r} \prod_{h=1}^{l} M_{h}^{k}\left(z_{h}(\mathbf{x}(t))\right)},
$$

are the normalized grades of membership being nonlinear functions of $\mathbf{x}(t)$. Note that due to Definition (2.4), we have $0 \leq p_{i}(\mathbf{x}(t)) \leq 1$ for all $i$ and $\sum_{i=1}^{r} p_{i}(\mathbf{x}(t))=1$ that implies the overall fuzzy model is achieved by aggregating the polynomial system models. 


\subsection{Model Improvement}

Our aim is to find the nonlinear functions $p_{i}(\mathbf{x}(t))$ in (2.4) treated as the membership functions of polynomial fuzzy model presented in Section 2.1 in such a way that the mismatch between the nonlinear plant and the fuzzy model is minimized. As membership function information will be subsequently considered in SOS-based stability analysis, they must be in the form of polynomials. To achieve this, we use a Taylor series expansion in several variables to formulate the membership function $p_{i}(\mathbf{x}(t))$ in different sub-regions for $\mathbf{x}$. Defining a proper fitness function quantifying mismatch between the nonlinear plant and the fuzzy model, a GA is used to optimize the coefficients of Taylor series for each $p_{i}(\mathbf{x}(t))$ in the corresponding sub-region.

We consider the Taylor series of nonlinear function $p_{i}(\mathbf{x}(t))$ in sub-region $S_{k}$ as follows:

$$
\begin{array}{r}
p_{i}(\mathbf{x}(t))=p_{i}(\mathbf{a})+(\mathbf{x}-\mathbf{a})^{T} \nabla\left(p_{i}(\mathbf{a})\right)+\frac{1}{2 !}(\mathbf{x}-\mathbf{a})^{T}\left\{\nabla^{2}\left(p_{i}(\mathbf{a})\right)\right\}(\mathbf{x}-\mathbf{a})+\cdots, \\
i=1,2, \ldots, r, \text { for } \mathbf{x} \in S_{k}, k=1,2, \ldots, D,
\end{array}
$$

where $\nabla\left(p_{i}(\mathbf{a})\right)$ is the gradient of $p_{i}(\mathbf{x}(t))$ evaluated at $\mathbf{x}=\mathbf{a}$, and the $\nabla^{2}\left(p_{i}(\mathbf{a})\right)$ is the Hessian matrix. Also $D$ indicates the number of sub-regions $S_{k}(k=1, \ldots, D)$ of $\mathbf{x}$ in which corresponded $p_{i}(\mathbf{x}(t))$ are determined. Applying the multi-index notation and considering a finite number of terms $(T)$ in the Taylor series expansion, (2.5) can be rewritten as follows:

$$
p_{i}(\mathbf{x}(t))=\sum_{q=0}^{T} \frac{(\mathbf{x}-\mathbf{a})^{q}}{q !} \nabla^{q}\left(p_{i}(\mathbf{a})\right)=\sum_{q=0}^{T} \omega_{q}(\mathbf{x}-\mathbf{a})^{q},
$$

where

$$
\omega_{q}=\frac{\nabla^{q}\left(p_{i}(\mathbf{a})\right)}{q !}, \quad q=0,1,2, \ldots, T
$$

Note that the sub-regions and their number are predefined depending upon the operating range of state variables $\mathbf{x}(t)$ shown in (2.1). On the other hand, the more sub-regions we choose, the more accurate polynomial membership functions $p_{i}(\mathbf{x}(t))$ can be achieved. Choosing a sufficiently large number of sub-regions, the size of each sub-region will be reduced thus leading to higher computational burden. The number of sub-regions should be carefully selected.

In order to form the grades of polynomial membership functions, the coefficients $\omega_{q}$ have to be specified. Due to the large search area $\omega_{q}(q=1, \ldots, T)$, a GA is employed to find the Taylor series coefficients $\omega_{q}$ for all of the membership functions $p_{i}(\mathbf{x}(t))$ such that the mismatch between the original nonlinear plant and the corresponding fuzzy model is minimized. As the state variables show exact behavior of the system for all time $\left[t_{0}, \infty\right]$, the following fitness function is considered to describe a mismatch error to be minimized by the GA:

$$
\underset{\omega_{q}}{\operatorname{Min}}(\text { Mismatch Error })=\underset{\omega_{q}}{\operatorname{Min}}\left(\int_{t_{0}}^{\infty}\|\mathbf{x}(t)-\widehat{\mathbf{x}}(t)\|_{2} d t\right),
$$


where $\|\cdot\|_{2}$ is the Euclidean norm $\left(L_{2}\right.$-norm); $\mathbf{x}(t)$ and $\widehat{\mathbf{x}}(t)$ are the state variable vectors of the original system and fuzzy model in (2.1) and (2.3), respectively. In fact, the GA finds the polynomial membership functions such that the response of the original nonlinear plant and the polynomial fuzzy model exhibit the highest similarity of the state trajectories over a given time period.

In order to proceed with the GA optimization, a finite number of terms $(T)$ in Taylor series must be predefined. To extract the simplest form of the membership functions, the number of terms for each polynomial in (2.6) is initially set to zero. Afterward, the GA seeks the feasible solutions for the predefined values $T$ until the state trajectories of original nonlinear system and polynomial fuzzy model become matched. If the obtained polynomial grades of membership functions do not satisfy the mismatch error criterion between the original model and polynomial fuzzy model, the number of terms $(T)$ of polynomials is increased incrementally, and the searching process is resumed. To specify the search area, reduce the computational burden, and arrive at the simplest polynomials, the coefficients $\omega_{q}$ assume values in $[-1,1]$. Moreover, the operating range of state variables $\mathbf{x}(t)$ is divided into $D$ sub-regions to form the sub-regions $S_{k}(k=1, \ldots, D)$. Choosing the Taylor series evaluation point a in (2.6) as the center of each sub-region, the searching process can be started by GA after specification of its parameters such as population size, crossover type, and mutation rate which will be explained in details when applied to simulation examples.

\subsection{PDC Polynomial Fuzzy Controller}

Now based on the PDC technique [10], a polynomial fuzzy controller is constructed given the polynomial fuzzy model in (2.2). In this way, we complete the overall feedback loop as follows.

Rule $i$ of the controller:

$$
\begin{aligned}
& \text { if } z_{1}(\mathbf{x}(t)) \text { is } M_{1}^{i} \text { and } \ldots \text { and } z_{\tau}(\mathbf{x}(t)) \text { is } M_{\tau}^{i} \text {, } \\
& \text { then } \mathbf{u}(t)=-\mathbf{F}_{i}(\mathbf{x}(t)) \boldsymbol{X}(\mathbf{x}(t)), \quad i=1,2, \ldots, r .
\end{aligned}
$$

The overall FMB control is expressed as follows:

$$
\mathbf{u}(t)=-\sum_{i=1}^{r} p_{i}(\mathbf{x}(t)) \mathbf{F}_{i}(\mathbf{x}(t)) X(\mathbf{x}(t))
$$

From (2.3) and (2.10), the closed-loop system reads as follows:

$$
\dot{\mathbf{x}}(t)=\sum_{i=1}^{r} \sum_{j=1}^{r} p_{i}(\mathbf{x}(t)) p_{j}(\mathbf{x}(t))\left\{\mathbf{A}_{i}(\mathbf{x}(t))-\mathbf{B}_{i}(\mathbf{x}(t)) \mathbf{F}_{j}(\mathbf{x}(t))\right\} \mathcal{X}(\mathbf{x}(t)) .
$$

Note that if $\boldsymbol{X}(\mathbf{x}(t))=\mathbf{x}(t)$ and $\mathbf{A}_{i}, \mathbf{B}_{i}$, and $\mathbf{F}_{j}$ are constant matrices for all $i$ and $j$, then the polynomial system reduces to the canonical T-S FMB control system [10]. In this sense, the polynomial FMB control system forms a general representation of the model.

For the sake of brevity, in the remainder of the paper, $\mathbf{x}(t), \boldsymbol{x}(\mathbf{x}(t))$, and $p_{i}(\mathbf{x}(t))$ are denoted as $\mathbf{x}, \boldsymbol{X}(\mathbf{x})$, and $p_{i}(\mathbf{x})$, respectively. 


\subsection{Stability Analysis}

So far, the nonlinear plant was represented by the polynomial fuzzy modeling in (2.3), which was improved by tuning the membership functions. Besides, the polynomial fuzzy controller of (2.10) was designed to form the feedback loop, and the closed-loop FMB control system was obtained as given in (2.11). Considering the Lyapunov stability theory, the SOS-based stability conditions of designed FMB control system are proposed in this section. As we have already expressed the membership functions in the form of polynomials, the proposed stability conditions take these functions into consideration. The approach presented here relies on the SOS decomposition of multivariable polynomials $[43,44]$. Let us start with some prerequisites.

Definition 2.2 (see [45]). A multivariate polynomial $\mathbf{P}(\mathbf{x}), \mathbf{x} \in \mathbb{R}^{n}$ is a SOS if there exist polynomials $\Gamma_{1}(\mathbf{x}), \ldots, \Gamma_{\mathcal{K}}(\mathbf{x})$ such that $\mathbf{P}(\mathbf{x})=\sum_{i=1}^{\mathcal{K}} \Gamma_{i}^{2}(\mathbf{x})$.

It can be shown that such a polynomial is equivalent with a special quadratic form as stated in the following proposition.

Proposition 2.3 (see [43]). Let $\mathbf{P}(\mathbf{x})$ be a polynomial in $\mathbf{x} \in \mathbb{R}^{n}$ of degree 2 l. In addition, let $\boldsymbol{\varsigma}(\mathbf{x})$ be a column vector whose elements are all monomials in $\mathbf{x}$ of degree no greater than $l$. Then, $\mathbf{P}(\mathbf{x})$ is a SOS if and only if there exists a positive semidefinite matrix $\widehat{\mathbf{P}}(\mathbf{x})$ such that.

$$
\mathbf{P}(\mathbf{x})=\varsigma^{T}(\mathbf{x}) \mathbf{P}(\mathbf{x}) \varsigma(\mathbf{x}) .
$$

To investigate the stability of the polynomial fuzzy control system in (2.11), a polynomial Lyapunov function given in $[34,35]$ is employed as follows:

$$
V(\mathbf{x}(t))=\chi^{T}(\mathbf{x}(t)) \mathrm{G}^{-1}(\tilde{\mathbf{x}}(t)) \chi(\mathbf{x}(t)),
$$

where $\mathbf{G}(\tilde{x}(t)) \in R^{N \times N}$ is a symmetric positive-definite polynomial matrix, and $\tilde{\mathbf{x}}=\left[x_{e_{1}}\right.$, $\left.x_{e_{2}}, \ldots, x_{e_{s}}\right]$ is a vector to be selected such that $\mathrm{E}=\left[e_{1}, e_{2}, \ldots, e_{s}\right]$ includes the row indices of $\mathbf{B}_{i}(\mathbf{x}(t))$ whose corresponding row is equal to zero for all $i$. Moreover, let $\mathbf{A}_{i}^{e}(\mathbf{x}(t))$ and $\mathbf{B}_{i}^{e}(\mathbf{x}(t))$ denote the $e$ th row in $\mathbf{A}_{i}(\mathbf{x}(t))$ and $\mathbf{B}_{i}(\mathbf{x}(t))$, respectively. Subsequently, we have

$$
\mathbf{B}_{i}^{e}(\mathbf{x}(t))=0, \quad \text { for } e \in \mathbf{E} \text {. }
$$

It should be noted that this assumption is made to avoid occurrence of a nonconvex condition [43]. The goal is to find a state feedback controller $\mathbf{u}(\mathbf{x})=-\mathbf{F}(\mathbf{x}) \mathcal{X}(\mathbf{x})$ which renders the equilibrium of the nonlinear system stable. Based on the Lyapunov stability theory, if the $\mathbf{G}(\widetilde{\mathbf{x}})$ and $\mathbf{G}^{-1}(\widetilde{\mathbf{x}})$ are positive-definite matrices in $\widetilde{\mathbf{x}}$ and $\partial V(\mathbf{x}(t)) / \partial t \leq 0$ (equality holds when $\mathbf{x}=0$ ), then the equilibrium of polynomial FMB control system in (2.11) is asymptotically stable [43]. We have

$$
\dot{V}(t)=\sum_{i=1}^{r} \sum_{j=1}^{r} p_{i}(\mathbf{x}) p_{j}(\mathbf{x}) \mathbf{w}^{T}(\mathbf{x}) \mathbf{H}_{i j}(\mathbf{x}) \mathbf{w}(\mathbf{x}),
$$


where $\mathbf{w}(\mathbf{x})=\mathrm{G}^{-1}(\tilde{\mathbf{x}}) \boldsymbol{X}(\mathbf{x})$ and

$$
\begin{aligned}
\mathbf{H}_{i j}(\mathbf{x})= & \left(\mathbf{A}_{i}(\mathbf{x}) \mathbf{G}(\mathbf{x})+\mathbf{B}_{i}(\mathbf{x}) \mathbf{R}_{j}(\mathbf{x})\right)^{T} \mathbf{Q}^{T}(\mathbf{x}) \\
& +\mathbf{Q}(\mathbf{x})\left(\mathbf{A}_{i}(\mathbf{x}) \mathbf{G}(\mathbf{x})+\mathbf{B}_{i}(\mathbf{x}) \mathbf{R}_{j}(\mathbf{x})\right) \\
& -\sum_{e \in \mathbf{E}} \frac{\partial \mathbf{G}(\tilde{\mathbf{x}})}{\partial \mathbf{x}_{e}} \mathbf{A}_{i}^{e}(\mathbf{x}) \boldsymbol{X}(\mathbf{x}),
\end{aligned}
$$

in which

$$
\mathbf{R}_{j}(\mathbf{x})=\mathbf{F}_{j}(\mathbf{x}) \mathbf{G}(\mathbf{x}),
$$

and $\mathbf{Q}(\mathbf{x}(t))$ is a $N \times n$ polynomial matrix:

$$
\mathbf{Q}(\mathbf{x})=\frac{\partial \chi(\mathbf{x})}{\partial \mathbf{x}}
$$

As the SOS procedure is employed to derive the stability conditions, the membership functions shown in (2.15) are brought into the stability analysis. To deal with the continuous membership functions, they must be expressed in the polynomial forms. As the grades of membership functions of fuzzy model and controller $p_{i}(\mathbf{x})$ have been earlier formulated as multivariable polynomials, each product term $p_{i}(\mathbf{x}) p_{j}(\mathbf{x})$ in $(2.15)$ is a polynomial as follows:

$$
\Omega_{i j}^{S_{k}}(\mathbf{x})=p_{i}(\mathbf{x}) p_{j}(\mathbf{x})
$$

in the sub-region $S_{k}$, for $i=1, \ldots, r, j=1, \ldots, r, k=1, \ldots, D$, and (2.20) can be rewritten as

$$
\dot{V}(t)=\sum_{k=1}^{D} \sum_{i=1}^{r} \sum_{j=1}^{r} \Theta_{k}(\mathbf{x}) \mathbf{w}^{T}(\mathbf{x}) \Omega_{i j}^{S_{k}}(\mathbf{x}) \mathbf{H}_{i j}(\mathbf{x}) \mathbf{w}(\mathbf{x}),
$$

in which $\Theta_{k}$ is a scalar function in the form

$$
\Theta_{k}(\mathbf{x})=\left\{\begin{array}{ll}
1, & \mathbf{x} \in S_{k}, \\
0, & \mathbf{x} \notin S_{k},
\end{array} \quad k=1,2, \ldots, D .\right.
$$

Based on the Lyapunov stability theory and considering (2.20), the equilibrium of polynomial FMB control system (2.11) is asymptotically stable if the following inequalities are satisfied

$$
\sum_{i=1}^{r} \sum_{j=1}^{r} \Theta_{k}(\mathbf{x}) \mathbf{w}^{T}(\mathbf{x}) \Omega_{i j}^{s_{k}}(\mathbf{x}) \mathbf{H}_{i j}(\mathbf{x}) \mathbf{w}(\mathbf{x})<0
$$

which hold for all sub-regions $S_{k}, k=1, \ldots, D, i=1, \ldots, r$, and $j=1, \ldots, r$. 
Note that each inequality in (2.22) should be satisfied only in the corresponding sub-region. On the other hand, SOSTOOLS solve SOS problems without considering subregions. Hence, the polynomials are considered with global operating range during searching numerical solutions. Thus, this may lead to conservative results.

To eliminate the conservative nature of the method, we apply the $S$-procedure approach [46] to define some slack expressions $L_{S_{k}}(\mathbf{x})$ such that they satisfy the following requirements:

$$
\begin{aligned}
& L_{S_{k}}(\mathbf{x}) \leq 0, \quad \mathbf{x} \in S_{k}, \\
& L_{S_{k}}(\mathbf{x})>0, \quad \mathbf{x} \notin S_{k},
\end{aligned}
$$

The stability analysis of the polynomial fuzzy systems with the use of polynomial Lyapunov functions reduces to SOS problems [43]. The SOS problems can be solved via the SOSTOOLS and the SeDuMi [47]. Referring to Proposition 2.3, the results of stability analysis can be stated in the following theorem.

Theorem 2.4. The equilibrium of polynomial FMB control system in (2.11) is asymptotically stable if there exist symmetric polynomial matrix $\mathbf{G}(\widetilde{\mathbf{x}}) \in \mathbb{R}^{N \times N}$, polynomial matrices $\mathbf{R}_{j}(\mathbf{x}) \in \mathbb{R}^{m \times N}$, and polynomial scalar $\varphi(\mathbf{x})$ such that the following expressions are SOS for $i=1, \ldots, r, j=1, \ldots, r$, and $k=1, \ldots, D$ :

(1) $v^{T}\left(\mathbf{G}(\tilde{\mathbf{x}})-\xi_{1}(\mathbf{x}) \mathbf{I}\right) \mathcal{v}$

(2) $-v^{T}\left(\sum_{i=1}^{r} \sum_{j=1}^{r}\left(\Omega_{i j}^{s_{k}}(\mathbf{x}) \mathbf{H}_{i j}(\mathbf{x})\right)+\xi_{2}^{s_{k}}(\mathbf{x}) \mathbf{I}\right) v+\varphi(\mathbf{x}) v^{T} L_{s_{k}}(\mathbf{x}) v, \quad \forall s_{k}, k=1, \ldots, D$,

(3) $\varphi(\mathbf{x})$,

where $\mathbf{I}$ is an identity matrix, $v(t) \in \mathbb{R}^{n}$ is an arbitrary vector independent of $\mathbf{x}, \xi_{1}(\mathbf{x})>0, \xi_{2}^{\xi_{k}}(\mathbf{x})>0$ for $\mathbf{x} \neq 0$ are predefined polynomial scalars, and the state feedback vector gains can be computed by (2.17). As the SOS conditions given in (2.24) express, the information of membership function has been taken into stability analysis.

\section{Simulation Studies}

The purpose of this section is to demonstrate effectiveness of proposed improved polynomial FMB control system through two examples. Also, another example is included at the end of this section in order to compare the proposed method in this paper with previous polynomial fuzzy systems. The obtained results obviously illustrate the priority of the proposed improved polynomial FMB control methodology. The simulations were carried out with the use of SOSTOOLS [47]. 


\subsection{Example 1}

Consider a nonlinear system with stable equilibrium at $\mathbf{x}=0$ for $\mathbf{x}(t) \in(-3,3)$ :

$$
\begin{aligned}
& \dot{x}_{1}(t)=\left(-\frac{29}{2}+\frac{23}{2} \sin \left(x_{2}(t)\right)\right) x_{2}(t)-7 x_{1}(t), \\
& \dot{x}_{2}(t)=\left(-\frac{5}{2}+\frac{1}{2} \sin \left(x_{2}(t)\right)\right) x_{2}(t)+x_{1}(t) .
\end{aligned}
$$

Based on the sector nonlinearity [10], setting $\gamma=1, r=2, z_{1}=x_{1}$, and $\mathcal{X}(\mathbf{x}(t))=\left[1, x_{2}\right]^{T}$ in polynomial fuzzy model in (2.2), the dynamics of nonlinear system in (3.1) is represented by the two-rule fuzzy model as follows.

Rule 1: If $z_{1(t)}$ is $p_{1}\left(x_{1}(t)\right)$, then $\dot{\mathbf{x}}(t)=\mathbf{A}_{1}(\mathbf{x}) \boldsymbol{X}(\mathbf{x}(t))$.

Rule 2: If $z_{1(t)}$ is $p_{2}\left(x_{1}(t)\right)$, then $\dot{\mathbf{x}}(t)=\mathbf{A}_{2}(\mathbf{x}) \boldsymbol{X}(\mathbf{x}(t))$.

Note that the membership functions $p_{i}(\mathbf{x}(t))$ are assumed to be functions of state variable $x_{1}$ as the premise variable $z_{1}$ depends of $x_{1}$. Let us set the following state matrices:

$$
\mathbf{A}_{1}(\mathbf{x})=\left[\begin{array}{cc}
-7 x_{1} & -3 \\
x_{1} & -2
\end{array}\right], \quad \mathbf{A}_{2}(\mathbf{x})=\left[\begin{array}{cc}
-7 x_{1} & -26 \\
x_{1} & -3
\end{array}\right]
$$

Choosing $\mathbf{A}_{1}$ and $\mathbf{A}_{2}$ as the state-dependent polynomial matrices, the overall system dynamics are computed by (2.3). The goal is to find the membership functions $p_{1}(\mathbf{x})$ and $p_{2}(\mathbf{x})$ in polynomial forms in such a way that the mismatch error in (2.8) between the original nonlinear plant and the corresponding fuzzy model is minimized. Considering the form of the membership functions according to (2.5), the GA is utilized to find the coefficients of the Taylor series $\omega_{q} \in(-1,1)$ for functions $p_{1}(\mathbf{x})$ and $p_{2}(\mathbf{x})$. In this example, $\mathbf{x}(t) \in(-3,3)$ and $D=5$ for the number of sub-regions as shown in Table 1 . The evaluated points $(\mathbf{x}=\mathbf{a})$ in (2.5) are predefined as the center value in each sub-region. Note that we consider the same sub-regions and evaluated points for $p_{1}(\mathbf{x})$ and $p_{2}(\mathbf{x})$. Also, Table 1 shows the proper slack expressions $L_{s_{k}}(\mathbf{x})$ introduced in (2.23), defined on each sub-region, and needed for stability analysis.

The GA parameters, used to minimize the fitness function in (2.8), are given in Table 2. Two types of crossover, namely, single point and two point, are applied. In each case with 150 generations, two crossover rates of 50 percent and 60 percent, two population sizes of 100 and 200 , with two mutation rates of 3 percent and 6 percent are employed. Each generation takes about 9 seconds, and the GA optimization process with 150 generations was completed in about 20 minutes on a PC with 2.6 GHz Pentium IV processor and $256 \mathrm{MB}$ of RAM. Applying GA for some initial conditions $\left[x_{1}(0), x_{2}(0)\right]$, the optimal polynomial grades $p_{1}(\mathbf{x})$ and $p_{2}(\mathbf{x})$ of membership functions are obtained as shown in Table 1.

Figure 1 shows the membership functions for sub-regions $S_{1}$ and $S_{3}$.

Figure 2 depicts the state trajectories of the original nonlinear system (3.1) compared with the obtained polynomial fuzzy model for initial conditions $\left[x_{1}(0), x_{2}(0)\right]=[0.1,0.1]$ and $\left[x_{1}(0), x_{2}(0)\right]=[2,-1]$. As the results show, the improved polynomial fuzzy model can properly capture the dynamics of original plant. 
Table 1: Slack expressions defined on subregions and obtained polynomial membership functions for Example 1.

\begin{tabular}{lccc}
\hline $\begin{array}{l}\text { Subregion } \\
\left(S_{k}\right)\end{array}$ & $\begin{array}{c}\text { Slack expression } \\
L_{S_{k}}(\mathbf{x})\end{array}$ & $\begin{array}{c}\text { Membership function } \\
p_{1}\left(x_{1}\right)\end{array}$ & $\begin{array}{c}\text { Membership function } \\
p_{2}\left(x_{1}\right)\end{array}$ \\
\hline$S_{1}:-3<x_{1}<-1$ & $\left(x_{1}+3\right)\left(x_{1}+1\right)$ & $0.2454-0.2081\left(x_{1}+2\right)+$ & $0.7546+0.2081\left(x_{1}+2\right)-$ \\
& & $0.2273\left(x_{1}+2\right)^{2}$ & $0.2273\left(x_{1}+2\right)^{2}$ \\
$S_{2}:-2<x_{1}<0$ & $\left(x_{1}+2\right) x_{1}$ & $0.1793+0.2702\left(x_{1}+1\right)+$ & $0.8207-0.2702\left(x_{1}+1\right)-$ \\
& & $0.2104\left(x_{1}+1\right)^{2}$ & $0.2104\left(x_{1}+1\right)^{2}$ \\
$S_{3}:-1<x_{1}<1$ & $\left(x_{1}+1\right)\left(x_{1}-1\right)$ & $0.4895+0.2003 x_{1}-0.0833\left(x_{1}\right)^{2}$ & $0.5105-0.2003 x_{1}+0.0833\left(x_{1}\right)^{2}$ \\
$S_{4}: 0<x_{1}<2$ & $x_{1}\left(x_{1}-2\right)$ & $0.8201+0.2708\left(x_{1}-1\right)-$ & $0.1799-0.2708\left(x_{1}-1\right)+$ \\
& & $0.211\left(x_{1}-1\right)^{2}$ & $0.211\left(x_{1}-1\right)^{2}$ \\
$S_{5}: 1<x_{1}<3$ & $\left(x_{1}-1\right)\left(x_{1}-3\right)$ & $0.7539-0.2085\left(x_{1}-2\right)-$ & $0.2461+0.2085\left(x_{1}-2\right)+$ \\
& & $0.2277\left(x_{1}-2\right)^{2}$ & $0.2277\left(x_{1}-2\right)^{2}$ \\
\hline
\end{tabular}

Table 2: GA parameters.

\begin{tabular}{lccc}
\hline Crossover type & Population size & Crossover rate (\%) & Mutation rate (\%) \\
\hline \multirow{2}{*}{ Single-point } & 100 & 50 & 3 \\
& 200 & 60 & 6 \\
\hline \multirow{2}{*}{ Two-point } & 100 & 50 & 3 \\
& 200 & 60 & 6 \\
\hline
\end{tabular}

The SOS-based stability conditions, proposed in Theorem 2.4, are satisfied, and a 6th order polynomial Lyapunov function was found as follows:

$$
\begin{aligned}
V(\mathbf{x}(t))= & 1.6218 x_{1}^{6}+0.5845 x_{1}^{5} x_{2}+0.8741 x_{1}^{4} x_{2}^{2}+0.3189 x_{1}^{3} x_{2}^{3}+0.3614 x_{1}^{2} x_{2}^{4} \\
& -0.03698 x_{1} x_{2}^{5}+0.0617 x_{2}^{6} .
\end{aligned}
$$

\subsection{Example 2}

Consider a nonlinear system with unstable equilibrium at $\mathbf{x}=0$ for $\mathbf{x}(t) \in(-10,10)$ :

$$
\begin{aligned}
& \dot{x}_{1}(t)=-x_{1}+x_{1}^{2}+x_{1}^{3}+x_{1}^{2} x_{2}-x_{1} x_{2}^{2}+x_{2}+x_{1} u, \\
& \dot{x}_{2}(t)=-(1+0.2763) \sin \left(x_{1}\right)-2 x_{2}+u .
\end{aligned}
$$

Similarly to the previous example, the dynamics of the nonlinear system in (3.4) is represented by three rules in the form of the polynomial fuzzy model in (2.2), where $\gamma=1$, $r=3, z_{1}=x_{1}$, and $\boldsymbol{X}(\mathbf{x}(t))=\left[x_{1}, x_{2}\right]^{T}$ as follows.

Rule 1: If $z_{1}(t)$ is $p_{1}\left(x_{1}(t)\right)$, then $\dot{\mathbf{x}}(t)=\mathbf{A}_{1}(\mathbf{x}) \boldsymbol{X}(\mathbf{x}(t))+\mathbf{B}_{1}(\mathbf{x}) \boldsymbol{u}(t)$.

Rule 2: If $z_{1}(t)$ is $p_{2}\left(x_{1}(t)\right)$, then $\dot{\mathbf{x}}(t)=\mathbf{A}_{2}(\mathbf{x}) \mathcal{X}(\mathbf{x}(t))+\mathbf{B}_{2}(\mathbf{x}) u(t)$.

Rule 3: If $z_{1}(t)$ is $p_{3}\left(x_{1}(t)\right)$, then $\dot{\mathbf{x}}(t)=\mathbf{A}_{3}(\mathbf{x}) \boldsymbol{X}(\mathbf{x}(t))+\mathbf{B}_{3}(\mathbf{x}) u(t)$. 


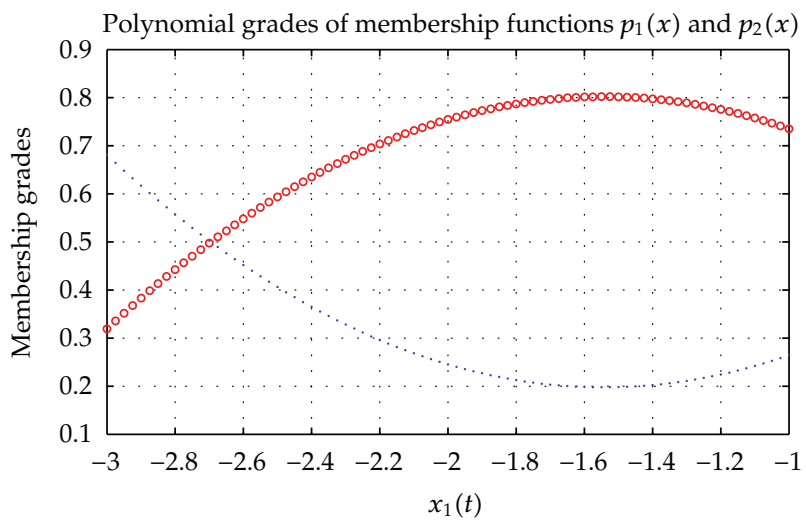

(a) sub-region $S_{1}, p_{1}:(\cdot)$ and $p_{2}:(\mathrm{o})$

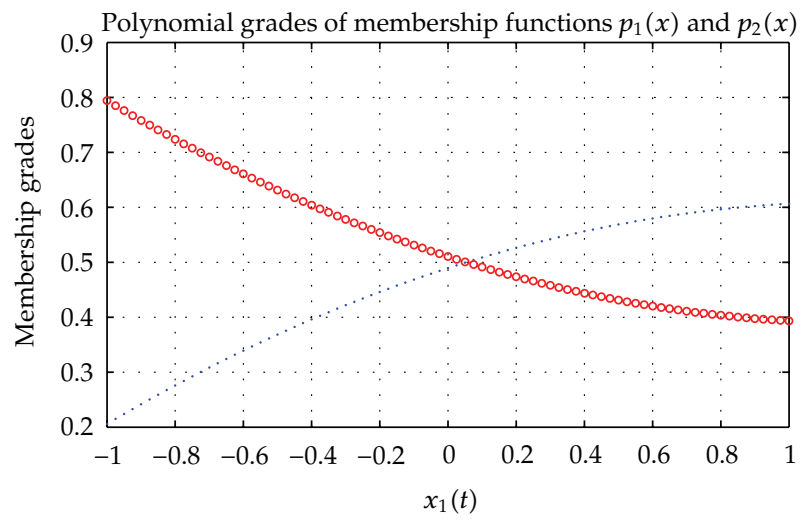

(b) sub-region $S_{3}, p_{1}:(\cdot)$ and $p_{2}:($ o)

Figure 1: Plots of membership functions, Example 1.

It should be noted that as the premise variable $z_{1}$ depends on the state variable $x_{1}$, then the membership functions $p_{i}(\mathbf{x})$ are considered as the functions of $x_{1}$. Let us assign the following matrices:

$$
\begin{array}{ll}
\mathbf{A}_{1}(\mathbf{x})=\left[\begin{array}{cc}
-1+x_{1}+x_{1}^{2}+x_{1} x_{2}-x_{2}^{2} & 1 \\
-1 & -2
\end{array}\right], & \mathbf{B}_{1}(\mathbf{x})=\left[\begin{array}{c}
x_{1} \\
1
\end{array}\right], \\
\mathbf{A}_{2}(\mathbf{x})=\left[\begin{array}{cc}
-1+x_{1}+x_{1}^{2}+x_{1} x_{2}-x_{2}^{2} & 1 \\
0 & -2
\end{array}\right], & \mathbf{B}_{2}(\mathbf{x})=\left[\begin{array}{c}
x_{1} \\
1
\end{array}\right], \\
\mathbf{A}_{3}(\mathbf{x})=\left[\begin{array}{cc}
-1+x_{1}+x_{1}^{2}+x_{1} x_{2}-x_{2}^{2} & 1 \\
0.2763 & -2
\end{array}\right], & \mathbf{B}_{3}(\mathbf{x})=\left[\begin{array}{c}
x_{1} \\
1
\end{array}\right] .
\end{array}
$$

As before, the membership functions are considered in the form of (2.5), and the GA is employed to find Taylor series coefficients $\omega_{q} \in(-1,1)$ for functions $p_{i}(\mathbf{x})(i=1,2,3)$. Furthermore, $\mathbf{x}(\mathrm{t}) \in(-10,10)$, and we choose $D=19$ to be the number of sub-regions as shown in Table 3. The position of the evaluated points $(\mathbf{x}=\mathbf{a})$ in (2.5) is the same as in 


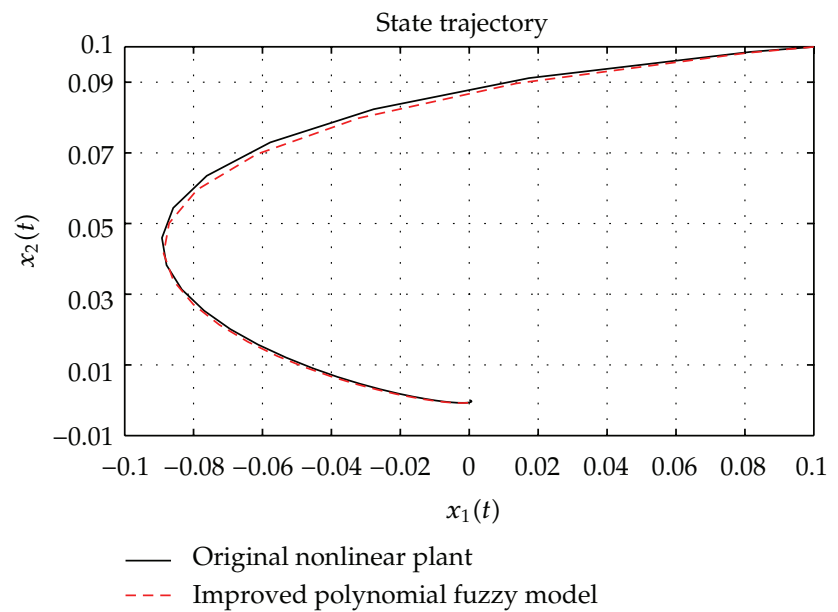

(a) $\left[x_{1}(0), x_{2}(0)\right]=[0.1,0.1]$

(a)

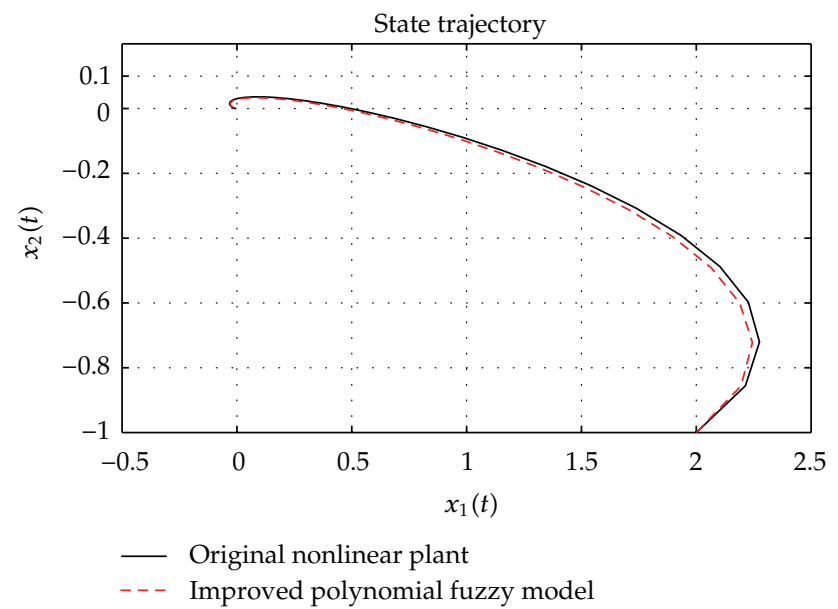

(b) $\left[x_{1}(0), x_{2}(0)\right]=[2,-1]$

(b)

Figure 2: State trajectories of original nonlinear plant and improved polynomial fuzzy model, Example 1.

Example 1. The same sub-regions and evaluated points are considered for all $p_{i}(\mathbf{x})$. Also, Table 3 shows the suitable slack expressions $L_{s_{k}}(\mathbf{x})$ in (2.23) defined on each sub-region required for stability analysis.

Applying GA with the parameters given in Table 2, the optimal polynomials $p_{i}(\mathbf{x})(i=$ $1,2,3)$ for different sub-regions are obtained as shown in Table 3. In this example, the GA optimization process that run for 650 generations was completed in about 90 minutes.

For instance, the plots of membership functions for sub-regions $S_{7}$ and $S_{15}$ are shown in Figure 3. 


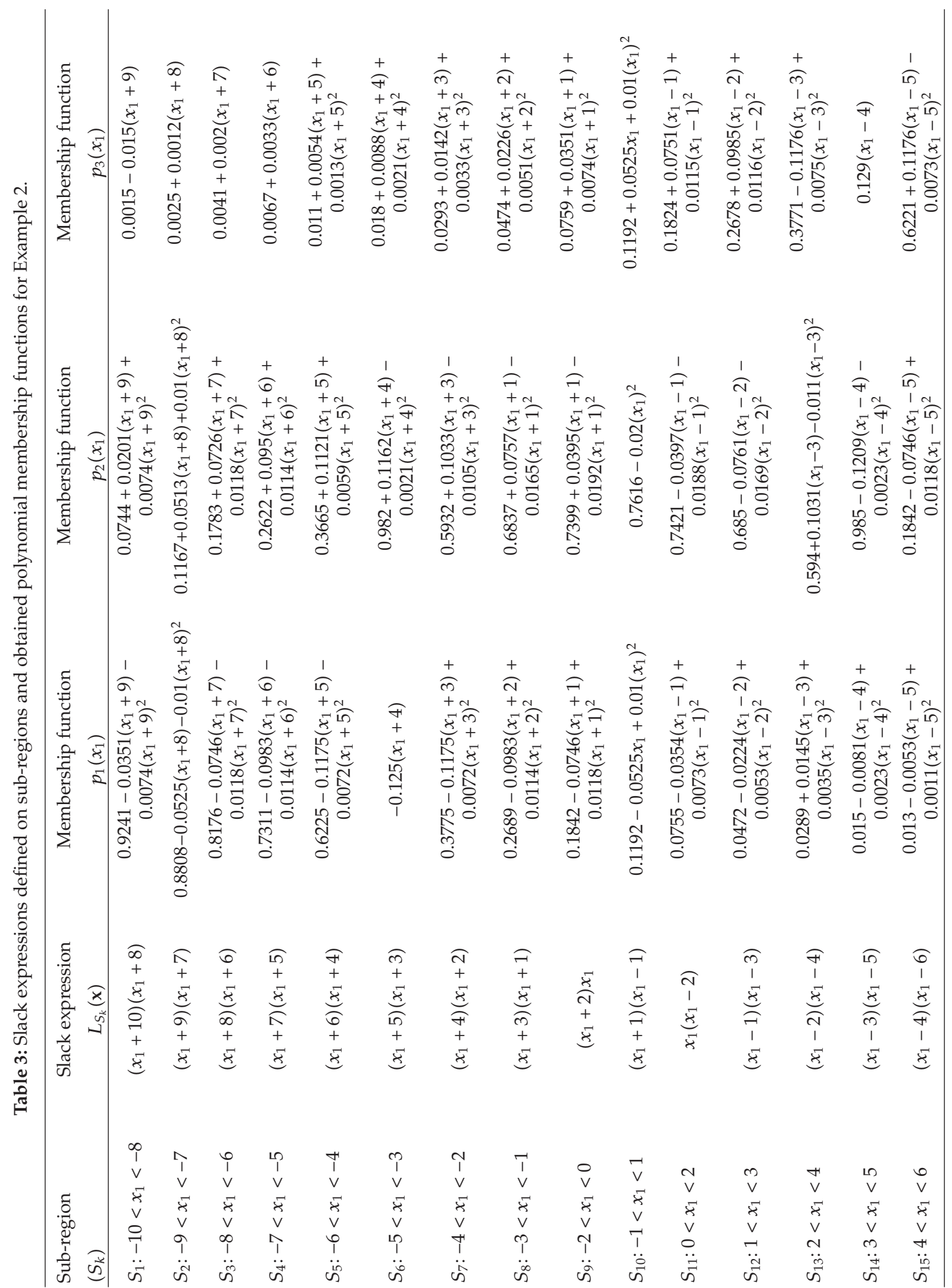




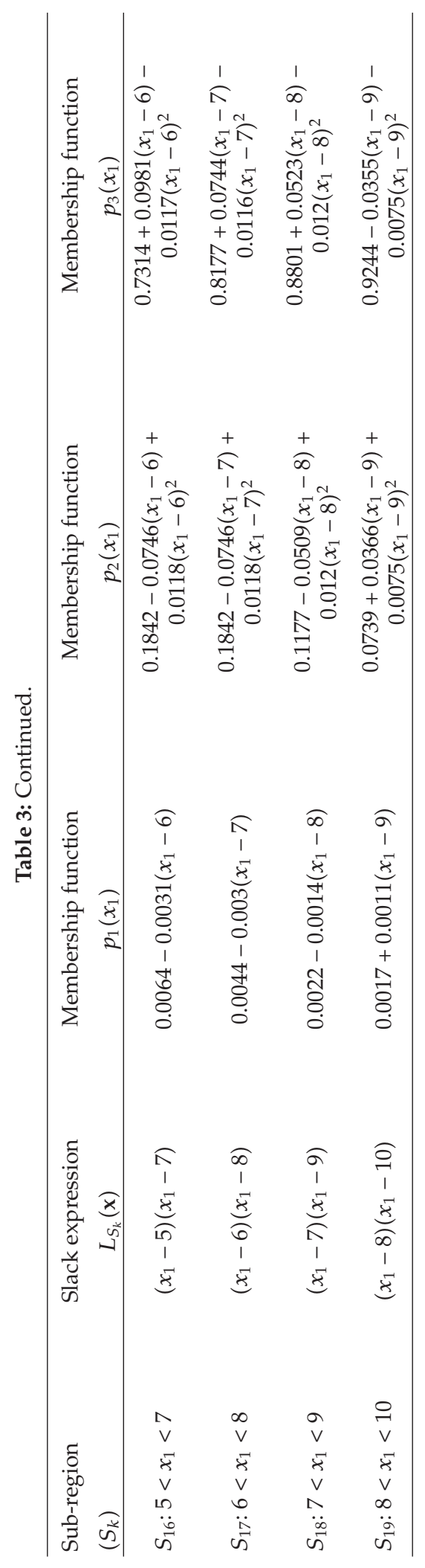




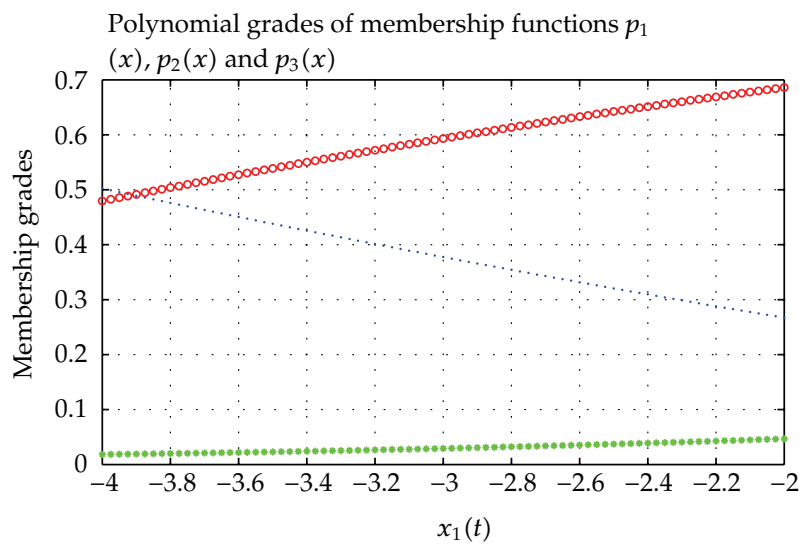

(a) sub-region $S_{7}, p_{1}:(\cdot), p_{2}:\left(\right.$ o) and $p_{3}:(*)$

Polynomial grades of membership functions $p_{1}$

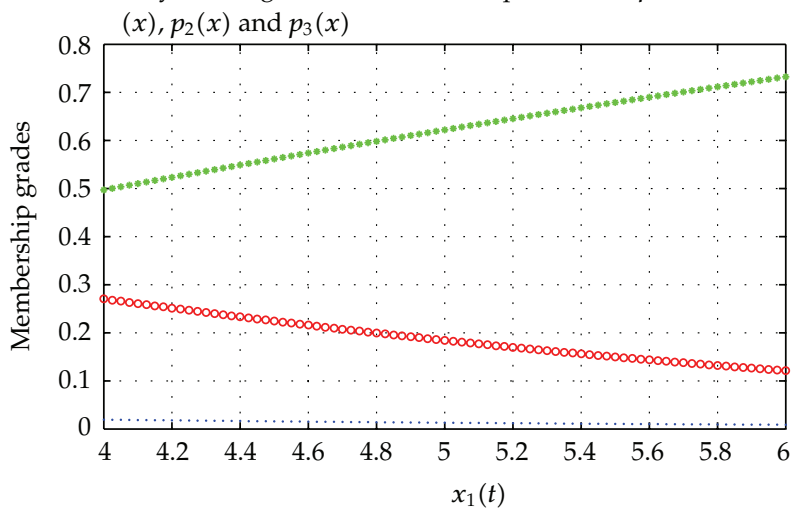

(b) sub-region $S_{15}, p_{1}:(\cdot), p_{2}:\left(\right.$ o) and $p_{3}:(*)$

Figure 3: Plots of membership functions, Example 2.

Based on the proposed stability conditions (Theorem 2.4) and considering slack expressions in Table 3, the feedback gains $\mathbf{F}_{i}(i=1,2,3)$ are calculated to close the feedback loop as follows:

$$
\begin{aligned}
& \mathbf{F}_{1}(\mathbf{x})=\left[\begin{array}{ll}
1.7705+1.2357 x_{1}+0.0199 x_{2} & -0.6998-0.3161 \times 10^{-4} x_{1}
\end{array}\right], \\
& \mathbf{F}_{2}(\mathbf{x})=\left[\begin{array}{ll}
2.6563+1.7418 x_{1}+0.0179 x_{2} & -0.6998-0.0155 \times 10^{-4} x_{1}
\end{array}\right], \\
& \mathbf{F}_{3}(\mathbf{x})=\left[\begin{array}{ll}
2.1214+1.7285 x_{1}+0.0168 x_{2} & -0.7504+0.3539 \times 10^{-4} x_{1}
\end{array}\right] .
\end{aligned}
$$

The SOS-based stability conditions are satisfied, and a quadratic polynomial Lyapunov function was found as follows:

$$
V(\mathbf{x}(t))=2.0960 x_{1}^{2}-0.1091 \times 10^{-5} x_{1} x_{2}+0.1078 x_{2}^{2} .
$$


Figure 4 displays the state trajectories of the original nonlinear system (3.4) when compared with the obtained polynomial fuzzy model for initial conditions $\left[x_{1}(0), x_{2}(0)\right]=$ $[-6,4]$ and $\left[x_{1}(0), x_{2}(0)\right]=[3.5,-5]$. Note that state trajectories indicate that the proposed fuzzy PDC controller in (2.10) can stabilize both nonlinear plant (3.4) and the closed-loop FMB control system in (2.11).

It should be noted that the information of attained polynomial membership functions have been fully included in the stability analysis. In both examples, we choose $\xi_{1}(\mathbf{x})=\xi_{2}^{S_{k}}(\mathbf{x})=$ 0.001 for all $i, j$, and $k$. Moreover, in simulation Example 2, there are no zero rows in the input matrices $\mathbf{B}_{i}(\mathbf{x})$. Thus, $\mathbf{G}(\widetilde{\mathbf{x}}(t))$ has been selected as a constant matrix.

\subsection{Comparison Example}

Consider a nonlinear system modeled by a three-rule polynomial fuzzy approach with $\mathcal{X}(\mathbf{x}(t))=\left[x_{1}, x_{2}\right]^{T}$ as follows:

$$
\begin{aligned}
& \mathbf{A}_{1}(\mathbf{x})=\left[\begin{array}{cc}
1.5-0.1 x_{1}^{2} & -7.5-0.25 x_{1} \\
0.05 & -0.1
\end{array}\right], \quad \mathbf{B}_{1}(\mathbf{x})=\left[\begin{array}{c}
1-0.25 x_{1}+x_{1}^{2} \\
0
\end{array}\right], \\
& \mathbf{A}_{2}(\mathbf{x})=\left[\begin{array}{cc}
0.01-0.5 x_{1}^{2} & -5+x_{1} \\
0.5 & -0.2
\end{array}\right], \quad \mathbf{B}_{2}(\mathbf{x})=\left[\begin{array}{c}
8+0.5 x_{1} \\
0
\end{array}\right], \\
& \mathbf{A}_{3}(\mathbf{x})=\left[\begin{array}{cc}
-a+0.5 x_{1}-x_{1}^{2} & -5 \\
0 & 0.05
\end{array}\right], \quad \mathbf{B}_{3}(\mathbf{x})=\left[\begin{array}{c}
-b+5+x_{1}^{2} \\
-1
\end{array}\right]
\end{aligned}
$$

where $a$ and $b$ are constant system parameters. The chosen membership functions for the fuzzy model are as the following:

$$
\begin{aligned}
& f_{1}\left(x_{1}\right)=1-\frac{1}{1+e^{-\left(x_{1}+4\right)}} \\
& f_{2}\left(x_{1}\right)=1-f_{1}\left(x_{1}\right)-f_{3}\left(x_{1}\right) \\
& f_{3}\left(x_{1}\right)=\frac{1}{1+e^{-\left(x_{1}-4\right)}}
\end{aligned}
$$

Also a two-rule polynomial fuzzy controller is designed with membership function chosen as

$$
g_{1}\left(x_{1}\right)= \begin{cases}1, & \text { for } x_{1}<-10 \\ \frac{-x_{1}+10}{20}, & \text { for }-10<x_{1}<10 \\ 0, & \text { for } x_{1}>10\end{cases}
$$

and $g_{2}\left(x_{1}\right)=1-g_{1}\left(x_{1}\right)$.

We applied our strategy proposed in this paper to find the polynomial membership functions for the nonlinear system for various parameters $a$ and $b$ as $1.5 \leq a \leq 10.5$ and $5 \leq b \leq 48$. Based on the proposed stability conditions in (Theorem 2.4) and considering 


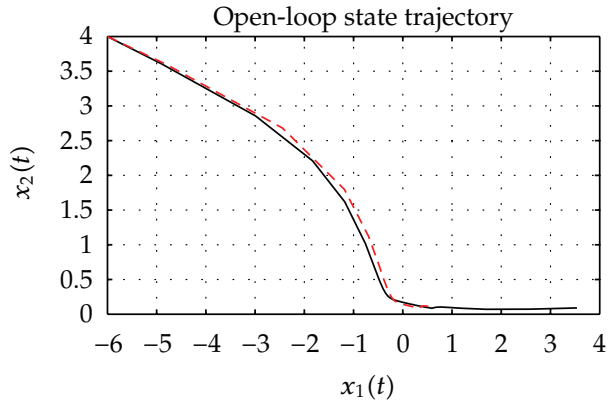

(a) open-loop system: $\left[x_{1}(0), x_{2}(0)\right]=[-6,4]$

(a)

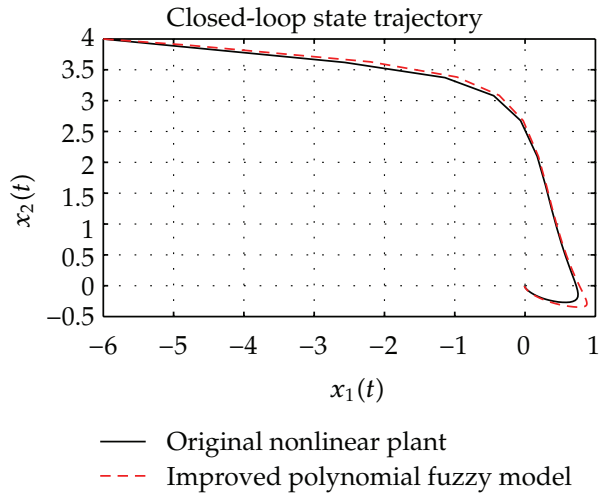

(c) closed-loop system: $\left[x_{1}(0), x_{2}(0)\right]=[-6,4]$

(c)

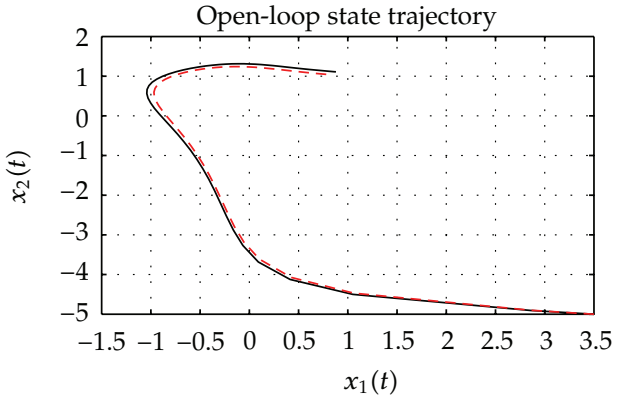

(b) open-loop system: $\left[x_{1}(0), x_{2}(0)\right]=[3.5,-5]$

(b)

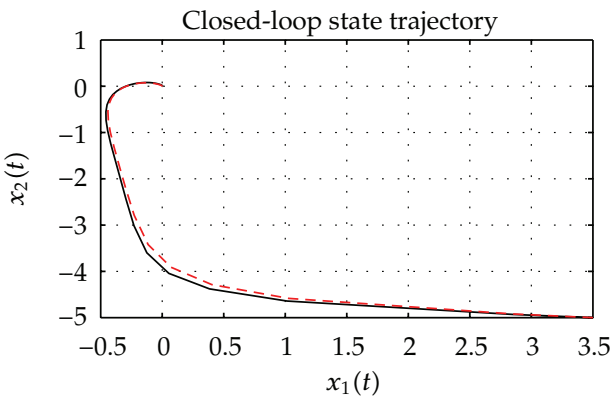

— Original nonlinear plant - - Improved polynomial fuzzy model

(d) closed-loop system: $\left[x_{1}(0), x_{2}(0)\right]=[3.5,-5]$

(d)

Figure 4: State trajectories of original nonlinear plant and improved polynomial fuzzy model for open-loop and closed-loop systems, Example 2.

obtained slack expressions, the feedback gains were computed to close the feedback loop. The SOS-based stability conditions were satisfied, and a 4 th order polynomial Lyapunov function was found.

Now, the stability conditions obtained in this paper are compared with those resulted in $[15,43]$ to check the stability region for the aforementioned intervals of $a$ and $b$. the stability regions given by $[15,43]$ and the proposed method are demonstrated in Figure 5. As this figure shows, the proposed stability conditions in (2.24) offer a larger stability region. Also, the stability conditions based on the LMI approach in [33] are used to check the system stability. But no stability region can be found.

\section{Discussion}

This paper has proposed a method based on Taylor-series to model nonlinear plants using polynomial fuzzy systems. By employing GA in an appropriate manner, the parameters of fuzzy model and controller including membership functions, rules, and relaxed stability conditions have been designed. It is noteworthy that the stability conditions derived in (2.24) are more relaxed than those resulted in previous works on polynomial fuzzy systems. On the other hand, our strategy to acquire these stability conditions is novel considering 


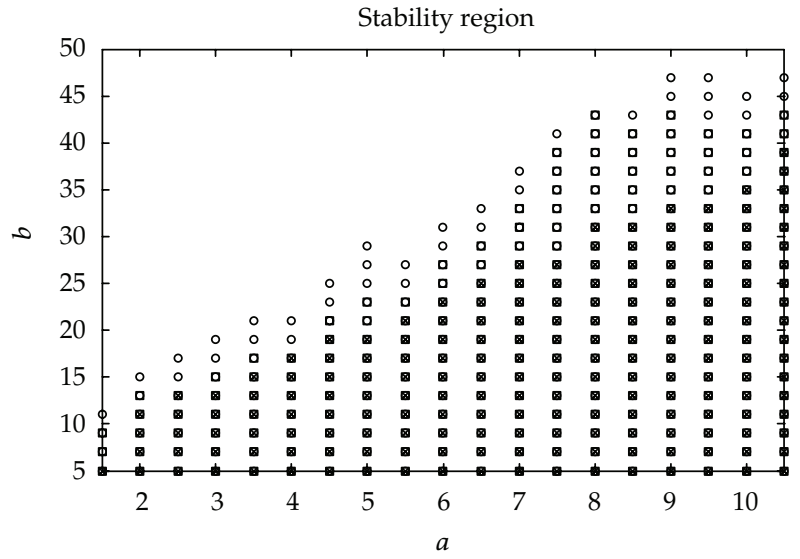

Figure 5: Stability regions obtained by conditions given in $[15,43]$ and Theorem 2.4 indicated by " $\square$ ", " $\times$ ", and " $\mathrm{o}$ ", respectively.

polynomial membership functions instead of conventional functions obtained by trial and error method depends on expert's knowledge. It is important to note that the effects of membership functions on stability are considered in analysis as shown in (2.15). As we use the polynomial Lyapunov function in (2.15) in stability analysis, expressing the membership functions in the form of polynomials is a worthwhile as well as essential task to achieve Theorem 2.4. Finally, in order to make it possible to use SOS approach to solve the obtained stability conditions, some slack expressions are defined in (2.23) based on the S-procedure method given in [46].

\section{Conclusion}

In this study, an improved polynomial fuzzy modeling for nonlinear dynamical systems is proposed via tuning the membership functions. Employing the Taylor series expansion, the membership functions in the corresponding sub-regions are constructed in the form of polynomials. Defining an appropriate fitness function, a GA is used to determine the coefficients of polynomials to minimize the mismatch error between the original nonlinear plant and polynomial fuzzy model. Furthermore, the proposed fuzzy modeling incorporates membership functions into the stability analysis and helps deriving SOS-based stability conditions for polynomial FMB control system. To form the FMB control system, a controller is designed based on the fuzzy PDC concept to close the feedback loop. To obtain more relaxed stability results, some slack expressions according to various sub-regions are considered in the SOS-based stability conditions. The solution to the SOS-based stability conditions can be numerically obtained by SOSTOOLS. The performance of proposed model, controller, and stability analysis is illustrated with the use of simulation examples.

\section{References}

[1] G. Feng, "A survey on analysis and design of model-based fuzzy control systems," IEEE Transactions on Fuzzy Systems, vol. 14, no. 5, pp. 676-697, 2006.

[2] K. Guelton, T. M. Guerra, M. Bernal, T. Bouarar, and N. Manamanni, "Comments on fuzzy control systems design via fuzzy lyapunov functions," IEEE Transactions on Systems, Man, and Cybernetics Part B, vol. 40, no. 3, pp. 970-972, 2010. 
[3] T. H. S. Li and S. H. Tsai, "T-S fuzzy bilinear model and fuzzy controller design for a class of nonlinear systems," IEEE Transactions on Fuzzy Systems, vol. 15, no. 3, pp. 494-506, 2007.

[4] T. Takagi and M. Sugeno, "Fuzzy identification of systems and its applications to modeling and control," IEEE Transactions on Systems, Man and Cybernetics, vol. 15, no. 1, pp. 116-132, 1985.

[5] C. W. Tao, Y. H. Chang, C. W. Chang, W. S. Chan, and J. S. Taur, "Robust and stable hybrid fuzzy control of a pendulum-cart system with particle swarm optimization," International Journal of Fuzzy Systems, vol. 12, no. 1, pp. 48-58, 2010.

[6] J. S. Chiou and S. H. Tsai, "Stability and stabilization of Takagi-Sugeno fuzzy switched system with time-delay," Journal of Systems and Control Engineering, vol. 226, no. 5, pp. 615-621, 2012.

[7] M. Sugeno and G. T. Kang, "Structure identification of fuzzy model," Fuzzy Sets and Systems, vol. 28, no. 1, pp. 15-33, 1988.

[8] G. Feng, "A survey on analysis and design of model-based fuzzy control systems," IEEE Transactions on Fuzzy Systems, vol. 14, no. 5, pp. 676-697, 2006.

[9] J. Adamy and A. Schwung, "Qualitative modeling of dynamical systems employing continuous-time recurrent fuzzy systems," Fuzzy Sets and Systems, vol. 161, no. 23, pp. 3026-3043, 2010.

[10] K. Tanaka and H. O. Wang, Fuzzy Control Systems Design and Analysis, John Wiley \& Sons, 2001.

[11] J. L. Castro, "Fuzzy logic controllers are universal approximators," IEEE Transactions on Systems, Man and Cybernetics, vol. 25, no. 4, pp. 629-635, 1995.

[12] H. K. Lam, "Polynomial fuzzy-model-based control systems: stability analysis via piecewise-linear membership functions," IEEE Transactions on Fuzzy Systems, vol. 19, no. 3, pp. 588-593, 2011.

[13] A. Sala and C. Ariño, "Asymptotically necessary and sufficient conditions for stability and performance in fuzzy control: applications of Polya's theorem," Fuzzy Sets and Systems, vol. 158, no. 24, pp. 2671-2686, 2007.

[14] H. K. Lam and M. Narimani, "Quadratic-stability analysis of fuzzy-model-based control systems using staircase membership functions," IEEE Transactions on Fuzzy Systems, vol. 18, no. 1, pp. 125$137,2010$.

[15] W. J. Chang, C. H. Chang, and C. C. Ku, "Fuzzy controller design for Takagi-Sugeno fuzzy models with multiplicative noises via relaxed non-quadratic stability analysis," Proceedings of the IME Part I: Journal of Systems and Control Engineering, vol. 224, no. 8, pp. 918-931, 2010.

[16] D. H. Lee, J. B. Park, and Y. H. Joo, "A new fuzzy lyapunov function for relaxed stability condition of continuous-time Takagi-Sugeno fuzzy systems," IEEE Transactions on Fuzzy Systems, vol. 19, no. 4, pp. 785-791, 2011.

[17] H. K. Lam and F. H. Leung, Stability Analysis of Fuzzy-Model-Based Control Systems, Springer, Berlin, Germany, 2011.

[18] H. Zhang and X. Xie, "Relaxed stability conditions for continuous-time TS fuzzy-control systems via augmented multi-indexed matrix approach," IEEE Transactions on Fuzzy Systems, vol. 19, no. 3, pp. 478-492, 2011.

[19] L. A. Mozelli, R. M. Palhares, and G. S. C. Avellar, "A systematic approach to improve multiple Lyapunov function stability and stabilization conditions for fuzzy systems," Information Sciences, vol. 179, no. 8, pp. 1149-1162, 2009.

[20] H. K. Lam, "LMI-based stability analysis for fuzzy-model-based control systems using artificial TS fuzzy model," IEEE Transactions on Fuzzy Systems, vol. 19, no. 3, pp. 505-513, 2011.

[21] J. Dong and G. H. Yang, "Control synthesis of T-S fuzzy systems based on a new control scheme," IEEE Transactions on Fuzzy Systems, vol. 19, no. 2, pp. 323-338, 2011.

[22] K. Tanaka and M. Sugeno, "Stability analysis and design of fuzzy control systems," Fuzzy Sets and Systems, vol. 45, no. 2, pp. 135-156, 1992.

[23] C. L. Chen, P. C. Chen, and C. K. Chen, "Analysis and design of fuzzy control system," Fuzzy Sets and Systems, vol. 57, no. 2, pp. 125-140, 1993.

[24] X. Huang, J. Cao, and Y. Li, "Takagi-Sugeno fuzzy-model-based control of hyperchaotic Chen system with norm-bounded uncertainties," Proceedings of the IME Part I: Journal of Systems and Control Engineering, vol. 224, no. 3, pp. 223-234, 2010.

[25] H. K. Lam and M. Narimani, "Stability analysis and perfomance design for fuzzy-model-based control system under imperfect premise matching," IEEE Transactions on Fuzzy Systems, vol. 17, no. 4, pp. 949-961, 2009.

[26] H. D. Tuan, P. Apkarian, T. Narikiyo, and Y. Yamamoto, "Parameterized linear matrix inequality techniques in fuzzy control system design," IEEE Transactions on Fuzzy Systems, vol. 9, no. 2, pp. 324-332, 2001. 
[27] X. Liu and Q. Zhang, "New approaches to Hœ controller designs based on fuzzy observers for T-S fuzzy systems via LMI," Automatica, vol. 39, no. 9, pp. 1571-1582, 2003.

[28] K. Tanaka, H. Ohtake, and H. O. Wang, "Guaranteed cost control of polynomial fuzzy systems via a sum of squares approach," IEEE Transactions on Systems, Man, and Cybernetics Part B, vol. 39, no. 2, pp. 561-567, 2009.

[29] Y. H. Chien, W. Y. Wang, Y. G. Leu, and T. T. Lee, "Robust adaptive controller design for a class of uncertain nonlinear systems using online T-S fuzzy-neural modeling approach," IEEE Transactions on Systems, Man, and Cybernetics Part B, vol. 41, no. 2, pp. 542-552, 2011.

[30] A. Lemos, W. Caminhas, and F. Gomide, "Multivariable gaussian evolving fuzzy modeling system," IEEE Transactions on Fuzzy Systems, vol. 19, no. 1, pp. 91-104, 2011.

[31] M. Chadli and H. Karimi, "Robust observer design for unknown inputs Takagi-Sugeno models," IEEE Transactions on Fuzzy Systems. In press.

[32] A. Schwung, T. Gußner, and J. Adamy, "Stability analysis of recurrent fuzzy systems: a hybrid system and sos approach," IEEE Transactions on Fuzzy Systems, vol. 19, no. 3, pp. 423-431, 2011.

[33] A. Sala and C. Ariño, "Relaxed stability and performance LMI conditions for Takagi-Sugeno fuzzy systems with polynomial constraints on membership function shapes," IEEE Transactions on Fuzzy Systems, vol. 16, no. 5, pp. 1328-1336, 2008.

[34] M. Narimani and H. K. Lam, "SOS-based stability analysis of polynomial fuzzy-model-based control systems via polynomial membership functions," IEEE Transactions on Fuzzy Systems, vol. 18, no. 5, pp. 862-871, 2010.

[35] A. Sala and T. M. Guerra, "Stability analysis of fuzzy systems: membership-shape and polynomial approaches," in Proceedings of the 17th World Congress, the International Federation of Automatic Control, pp. 5605-5610, Seoul, Republic of Korea, 2008.

[36] C. W. Park and Y. W. Cho, "T-S model based indirect adaptive fuzzy control using online parameter estimation," IEEE Transactions on Systems, Man, and Cybernetics Part B, vol. 34, no. 6, pp. 2293-2302, 2004.

[37] Y. J. Liu, W. Wang, S. C. Tong, and Y. S. Liu, "Robust adaptive tracking control for nonlinear systems based on bounds of fuzzy approximation parameters," IEEE Transactions on Systems, Man, and Cybernetics Part A, vol. 40, no. 1, pp. 170-184, 2010.

[38] M. Chadli and T. M. Guerra, "LMI solution for robust static output feedback control of Takagi-Sugeno fuzzy models," IEEE Trans. on Fuzzy Systems, vol. 20, no. 6, 2012.

[39] C. W. Park and M. Park, "Adaptive parameter estimator based on T-S fuzzy models and its applications to indirect adaptive fuzzy control design," Information Sciences, vol. 159, no. 1-2, pp. 125$139,2004$.

[40] Y. W. Cho, C. W. Park, and M. Park, "An indirect model reference adaptive fuzzy control for SISO Takagi-Sugeno model," Fuzzy Sets and Systems, vol. 131, no. 2, pp. 197-215, 2002.

[41] Y. W. Cho, C. W. Park, J. H. Kim, and M. Park, "Indirect model reference adaptive fuzzy control of dynamic fuzzy state-space model," IEE Proceedings, Control Theory and Applications, vol. 148, no. 4, pp. 273-282, 2001.

[42] T. J. Koo, "Stable model reference adaptive fuzzy control of a class of nonlinear systems," IEEE Transactions on Fuzzy Systems, vol. 9, no. 4, pp. 624-636, 2001.

[43] K. Tanaka, H. Yoshida, H. Ohtake, and H. O. Wang, "A sum-of-squares approach to modeling and control of nonlinear dynamical systems with polynomial fuzzy systems," IEEE Transactions on Fuzzy Systems, vol. 17, no. 4, pp. 911-922, 2009.

[44] S. Prajna, A. Papachristodoulou, and F. Wu, "Nonlinear control synthesis by sum of squares optimization: a Lyapunov-based approach," in Proc. Asian Control Conf., pp. 157-165, Melbourne, Australia, July 2004.

[45] A. Papachristodoulou and S. Prajna, "A tutorial on sum of squares techniques for systems analysis," in 2005 American Control Conference, ACC, pp. 2686-2700, Portland, Ore, USA, June 2005.

[46] S. P. Boyd, Linear Matrix Inequalities in System and Control Theory, Society for Industrial \& Applied, 1994.

[47] S. Prajna, A. Papachristodoulou, P. Seiler, and P. A. Parrilo, SOSTOOLS: Sum of Squares Optimization Toolbox for MATLAB, Version 2.00, California Institute of Technology, Pasadena, Calif, USA, 2004. 


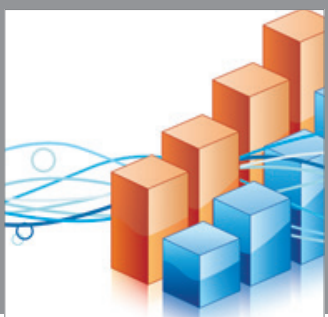

Advances in

Operations Research

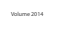

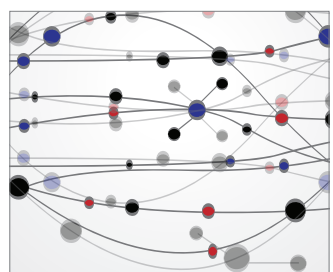

\section{The Scientific} World Journal
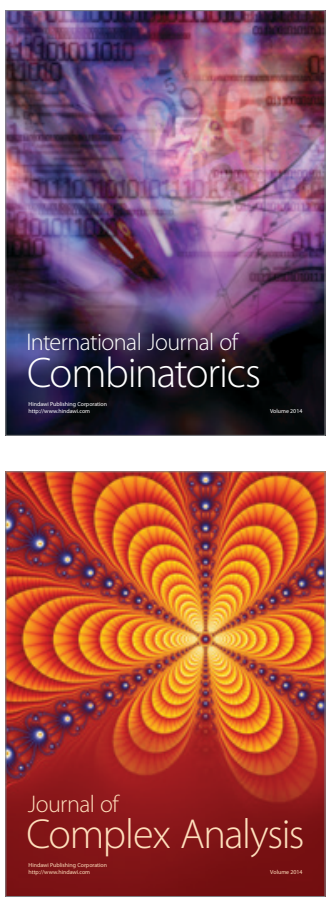

International Journal of

Mathematics and

Mathematical

Sciences
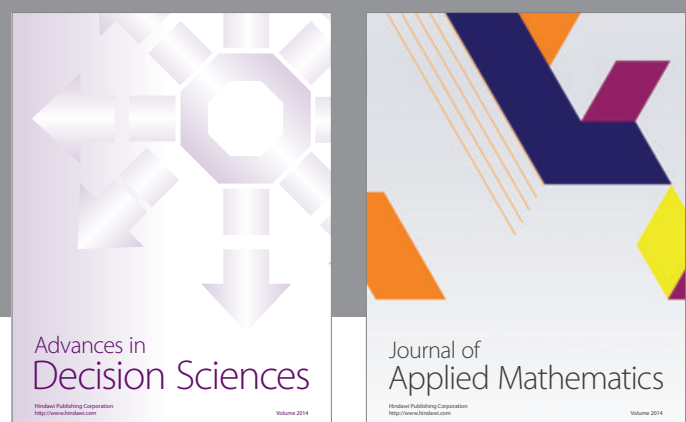

Journal of

Applied Mathematics
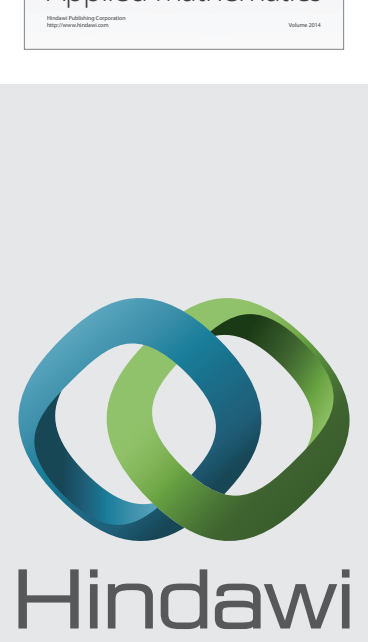

Submit your manuscripts at http://www.hindawi.com
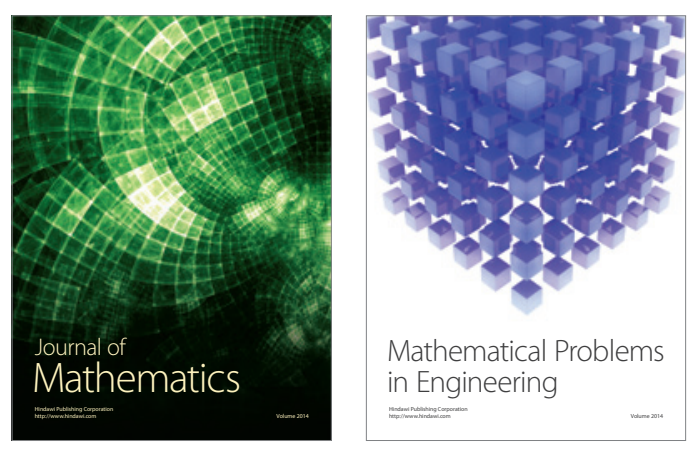

Mathematical Problems in Engineering
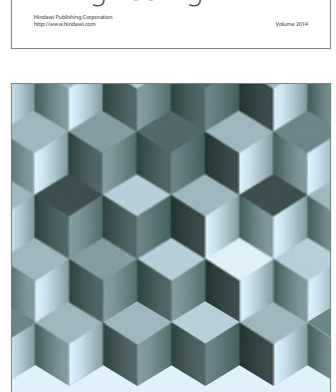

Journal of

Function Spaces
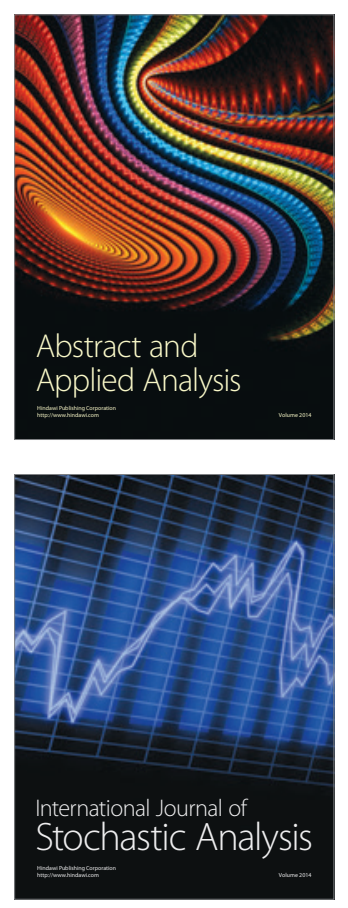

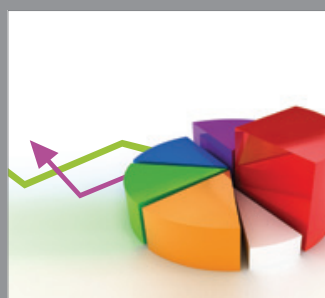

ournal of

Probability and Statistics

Promensencen
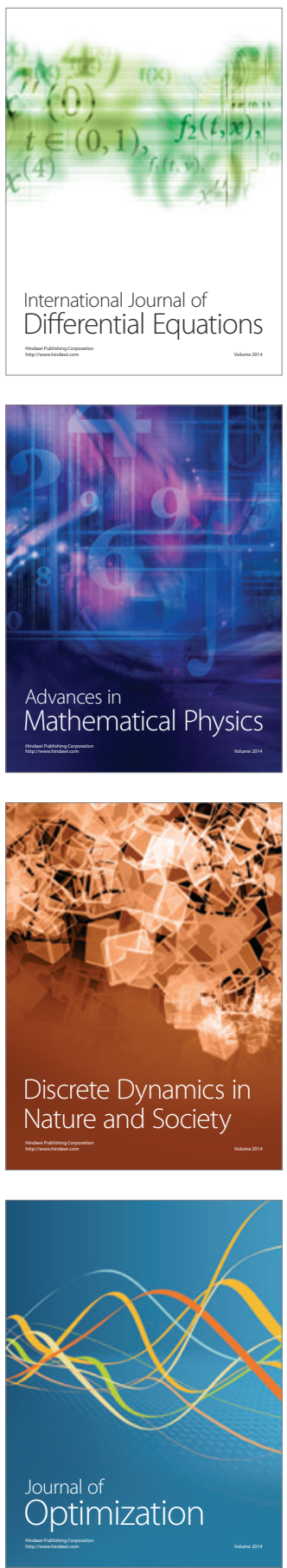